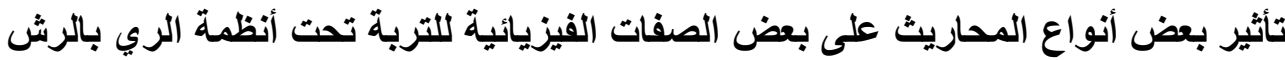

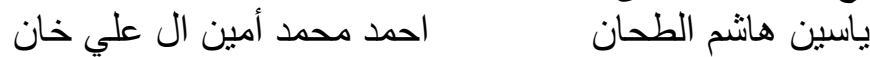 قسم المكنة الزراعية/ كلية الزراعة والغابات/ جامعة الموصل/ العر اق اق النين
}

\section{الخلاصة}

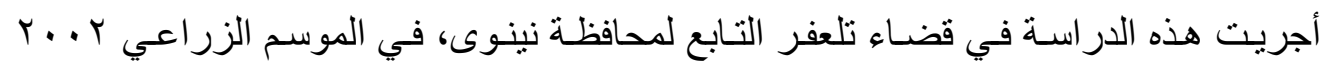

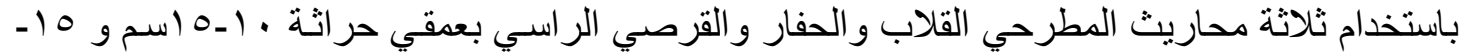

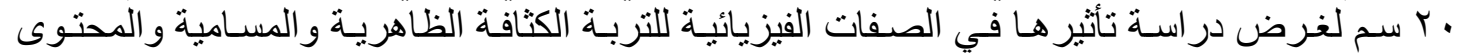

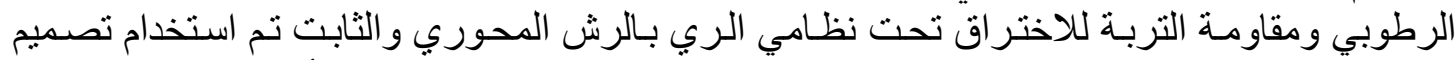

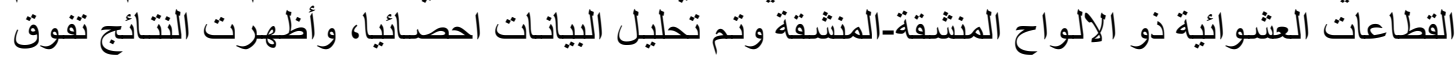

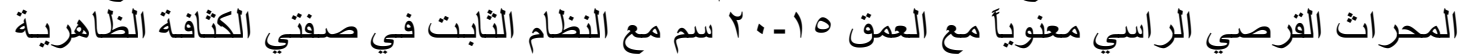

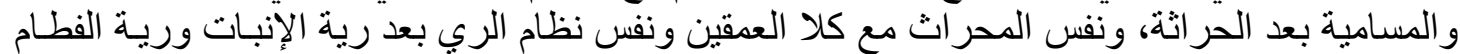

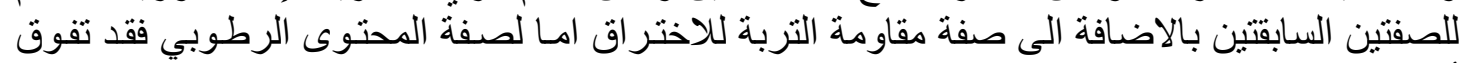

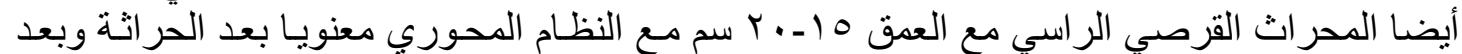

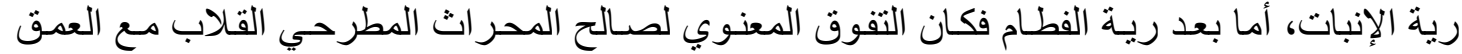

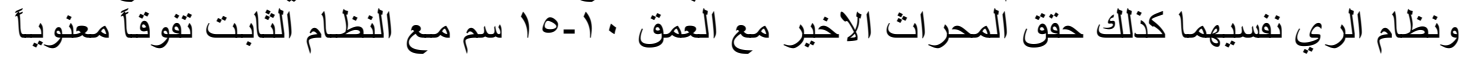

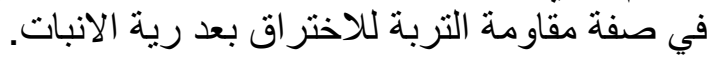

\section{المقدمة}

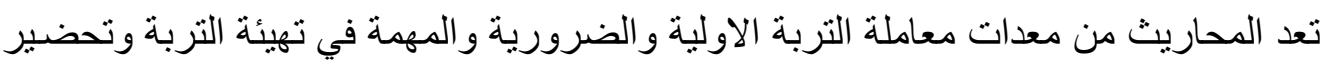

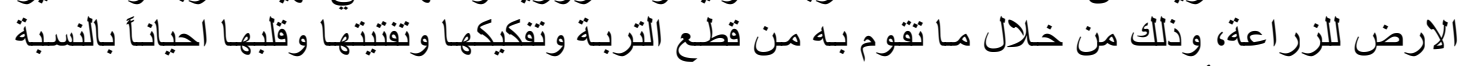

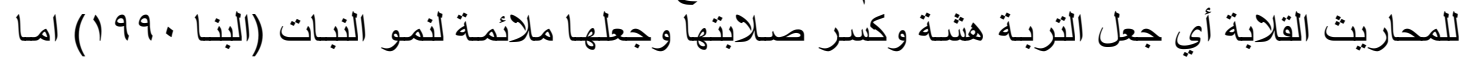

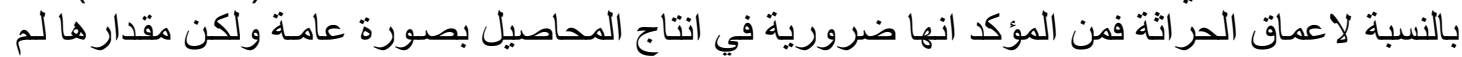

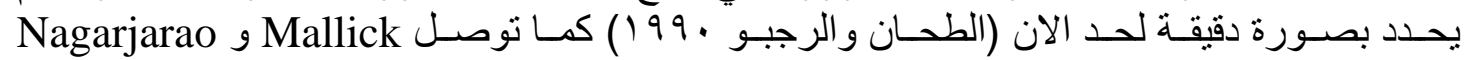

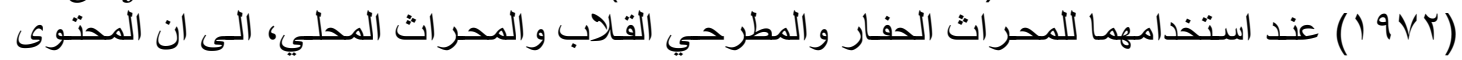

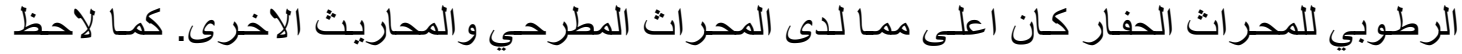
Henderson

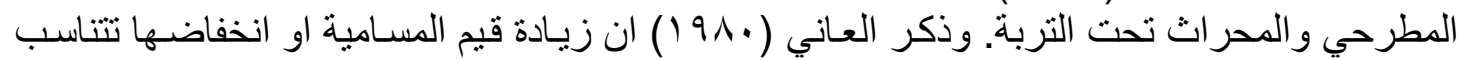

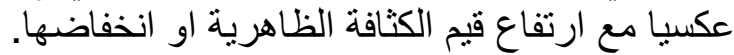

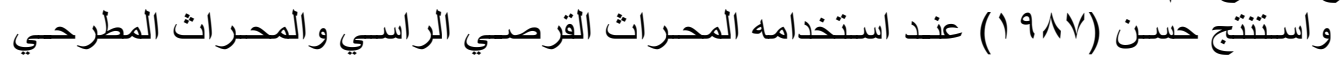

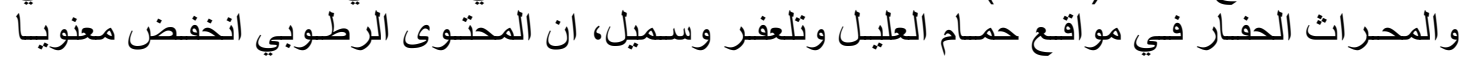

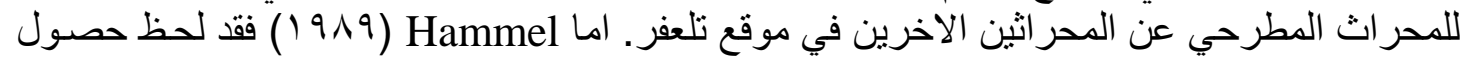

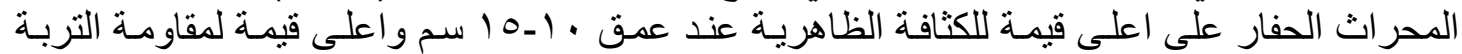

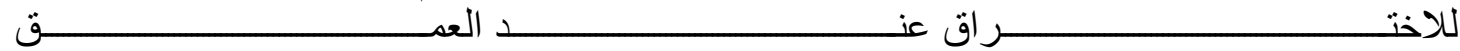

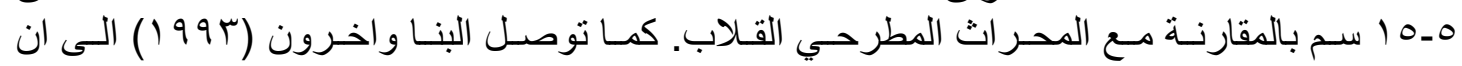

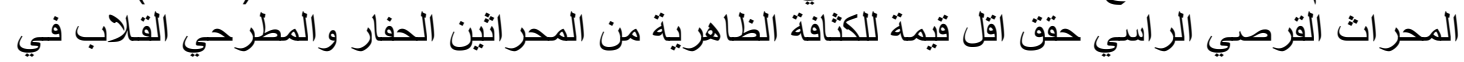

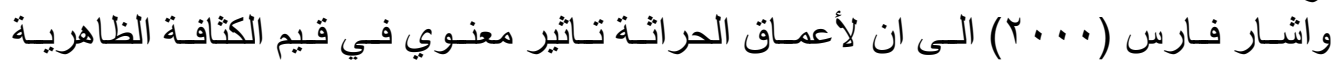

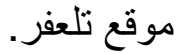

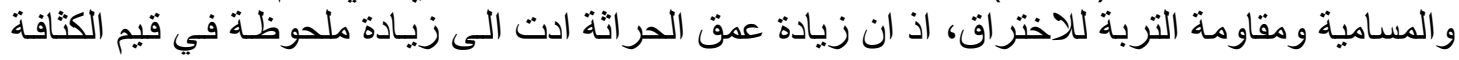

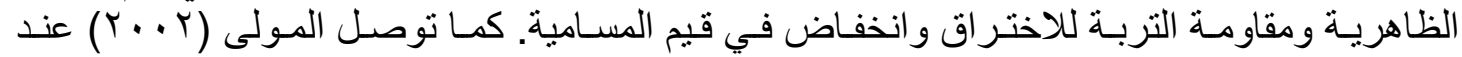

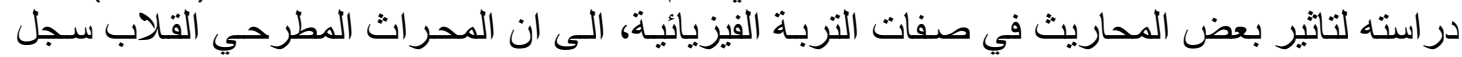

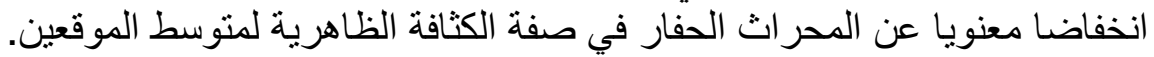

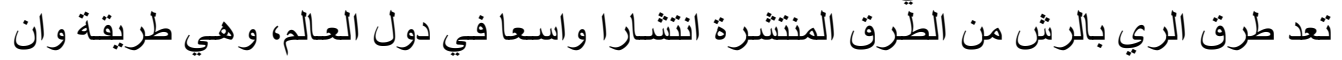

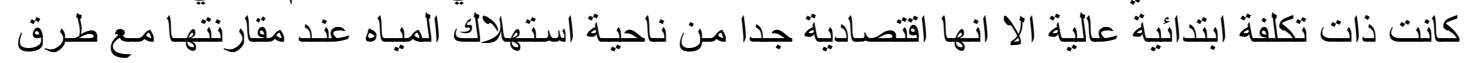

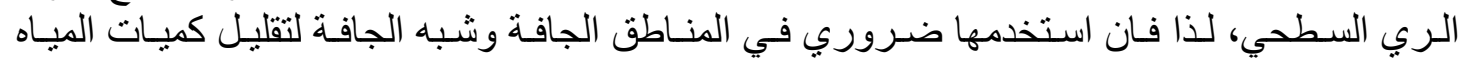
روفة 


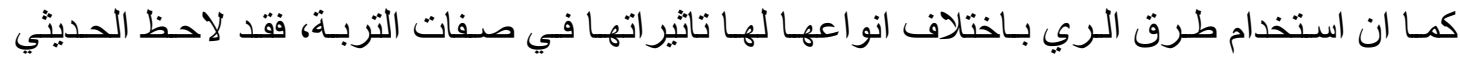

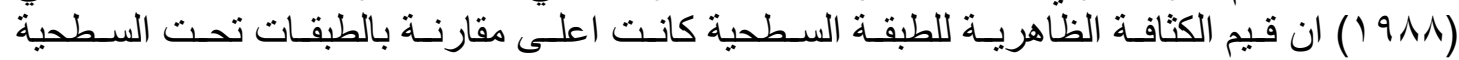

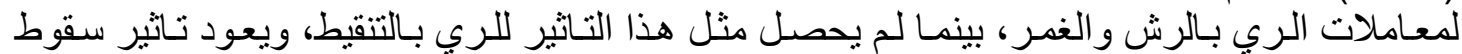

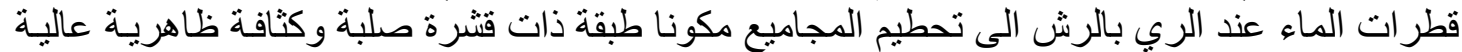

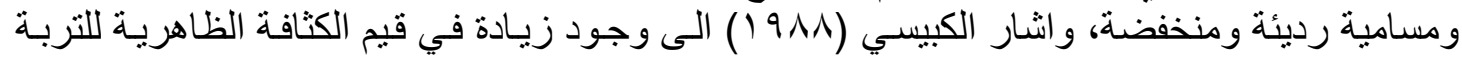

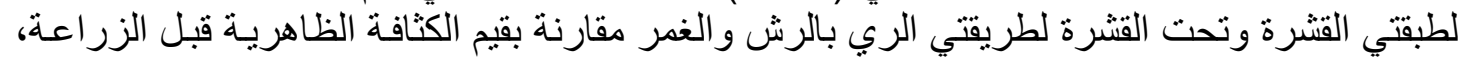

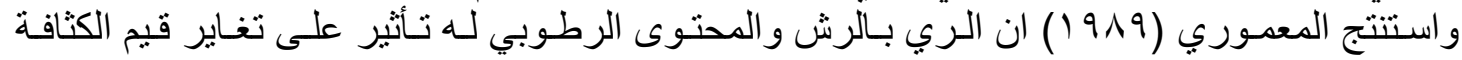

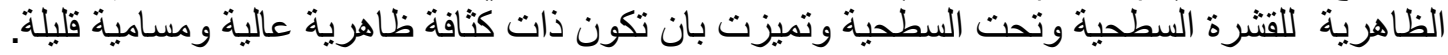

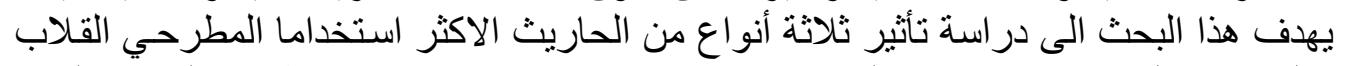

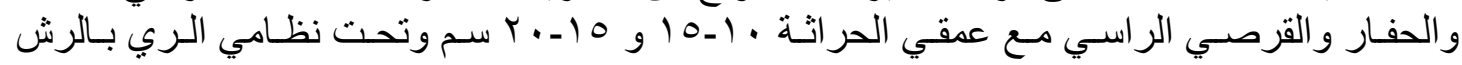

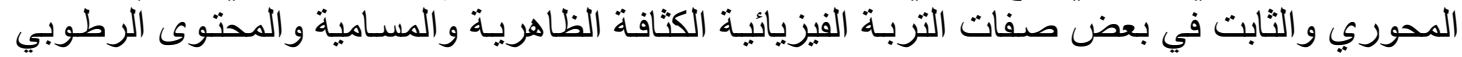
ومقاومة التربة للاختر اق وفي حقلين تمت زر اعتهما بمحصول الذرة الصفئ الصفراء.

\section{مواد البحث وطرائقه}

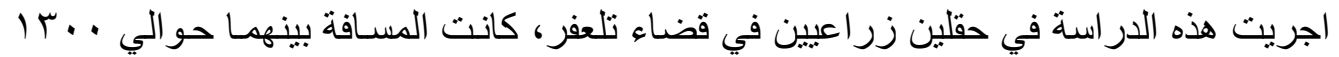

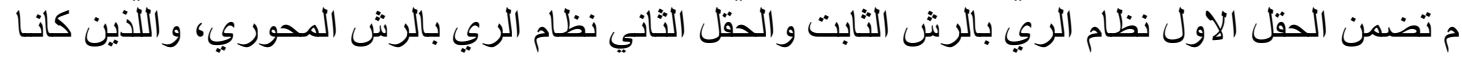

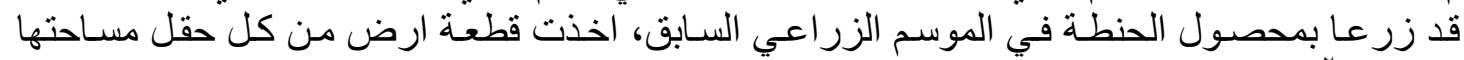

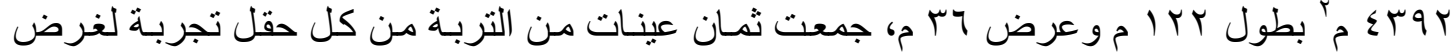

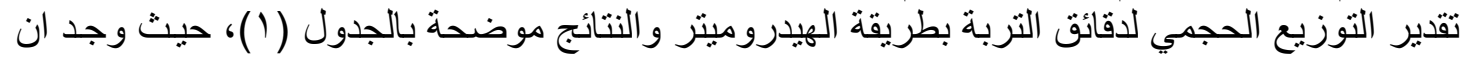

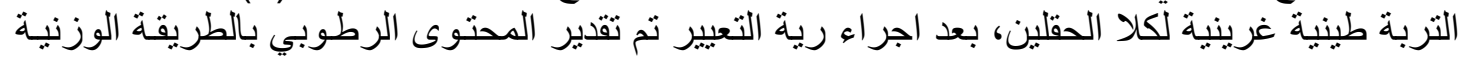

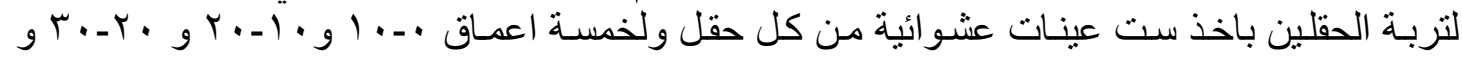

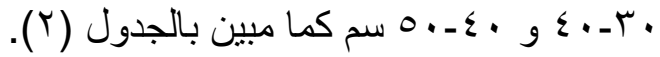

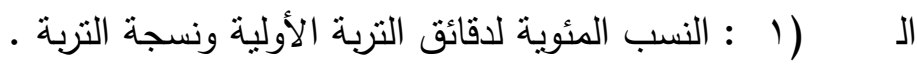

\begin{tabular}{|c|c|c|c|c|}
\hline نسجة التربة & نسبة الرمل (\%) & نسبة الغرين (\%) & نسبة الطين (\%) & حقلي التجربة \\
\hline طينية غرينية & 11.2 & § & $\varepsilon \vee .7$ & الحقل الأول \\
\hline طينية غرينية & $1 \cdot . \wedge$ & $\varepsilon r . V$ & $\leqslant 0.0$ & الحقل الثاني \\
\hline
\end{tabular}

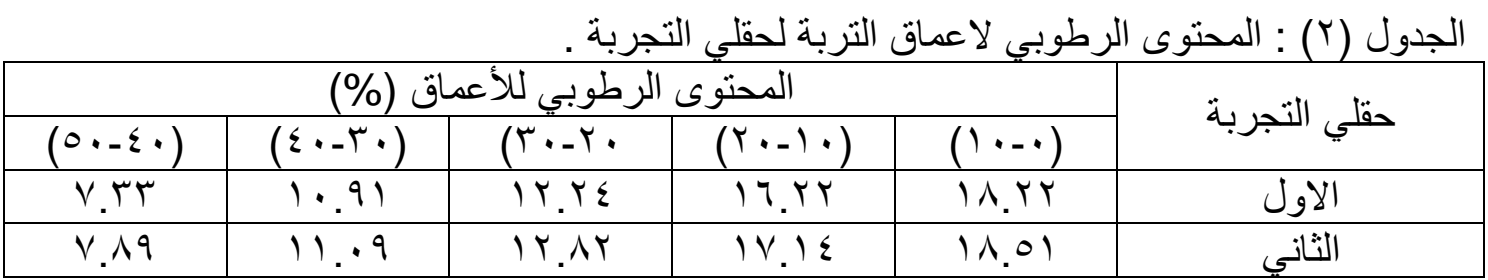

تم تقسيم الحقل وفق تصميم القطاعات العشو ائية الكاملة، واستخدمت طريقة الالواح (المنشقة.

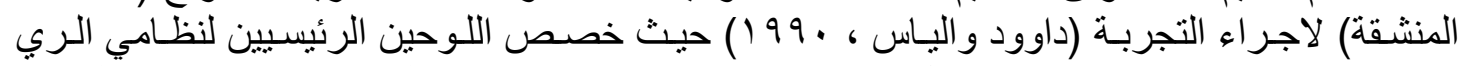

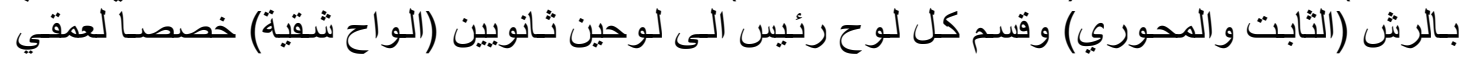

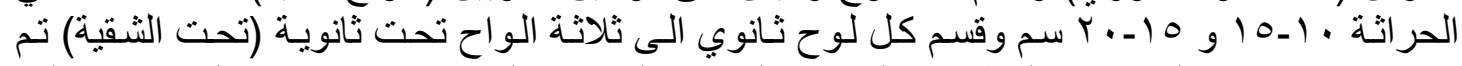

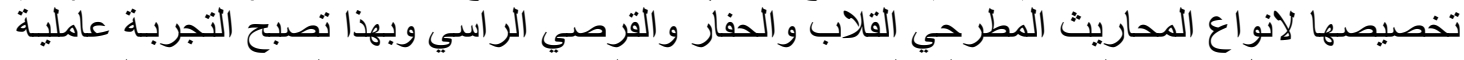

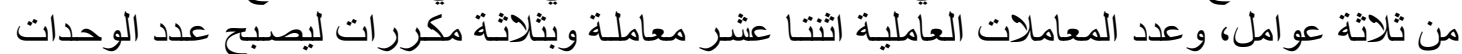

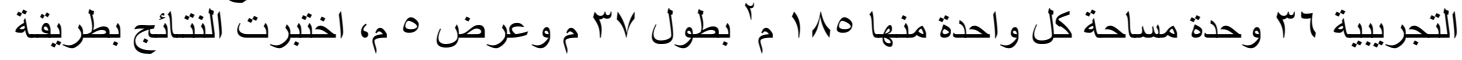

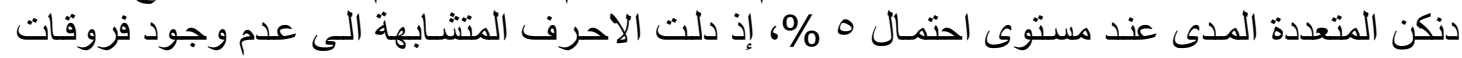

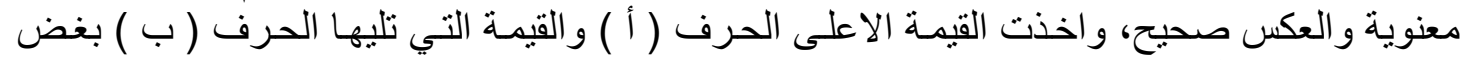

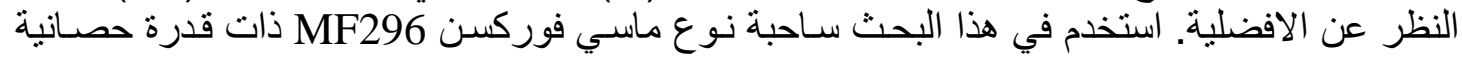

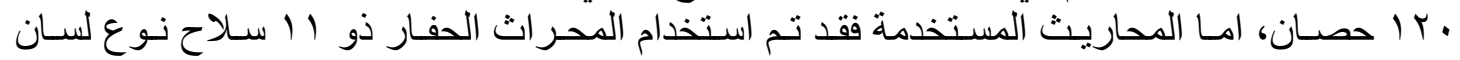




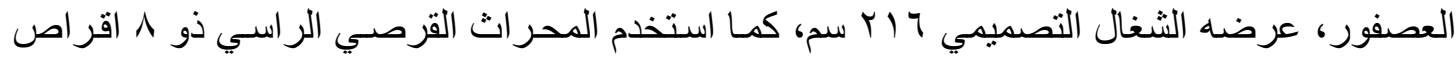

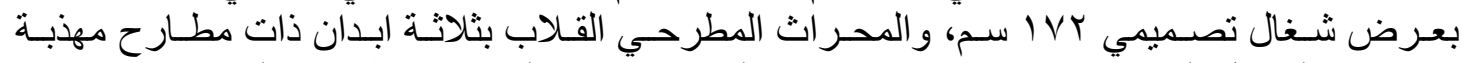

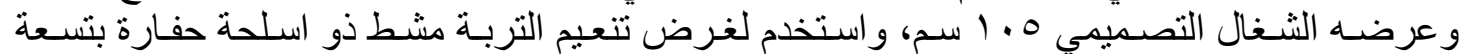

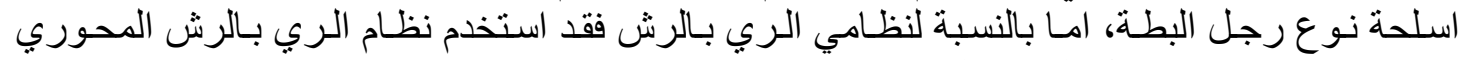

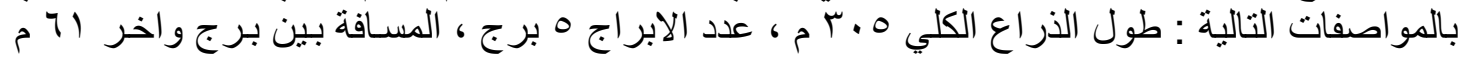

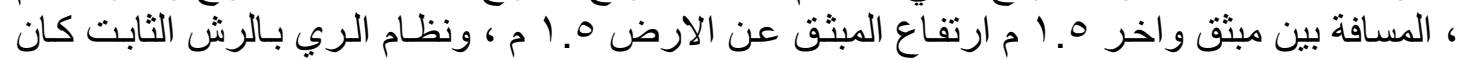

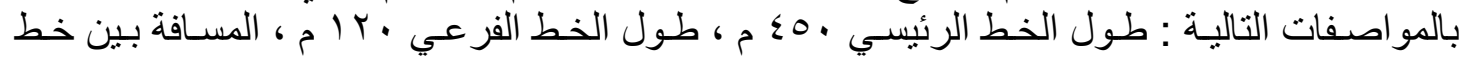

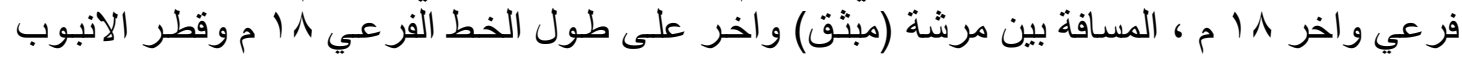

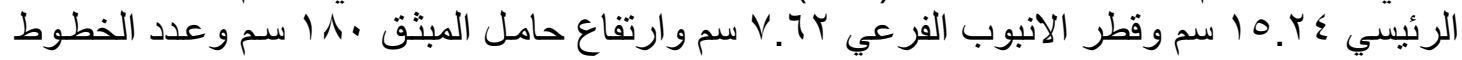

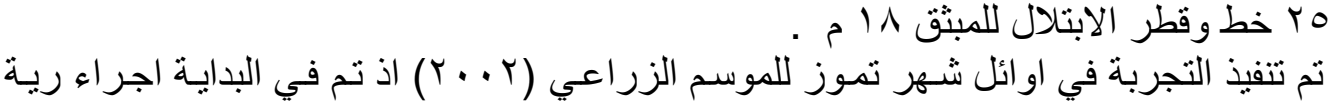

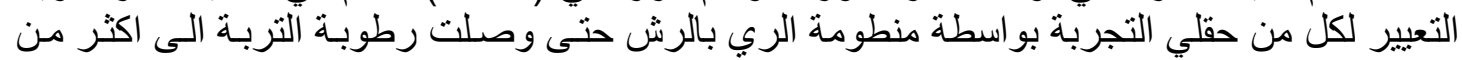

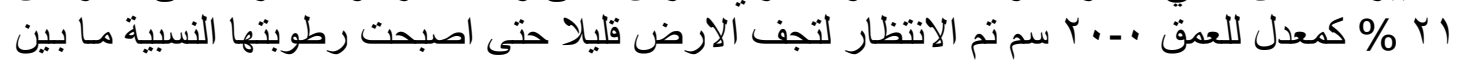

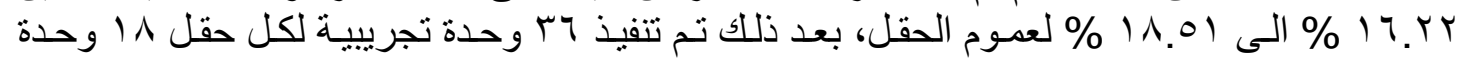

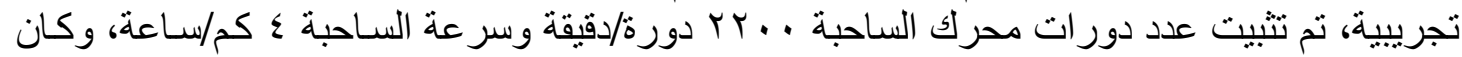

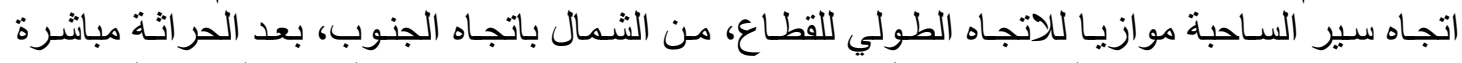

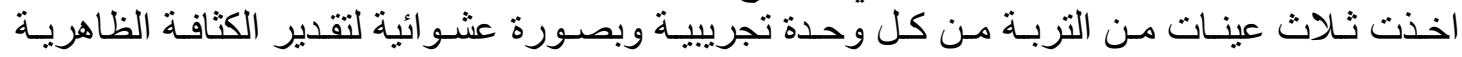

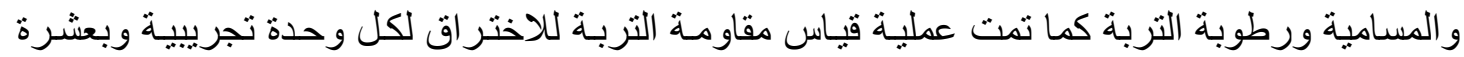

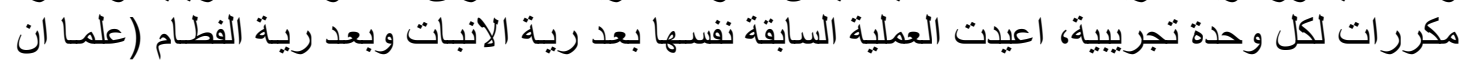

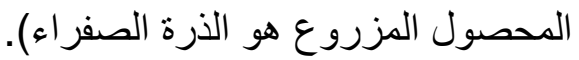

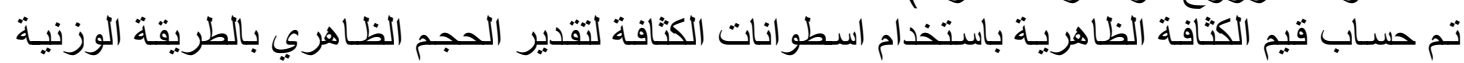

و استخدمت العلاقة التالية لتقدير قيم الكثافة الظاهرية وكما جاء في الحسن (.99 (1) و البنا (99 (199). $\mathrm{Pb}=\frac{M s}{V t}$

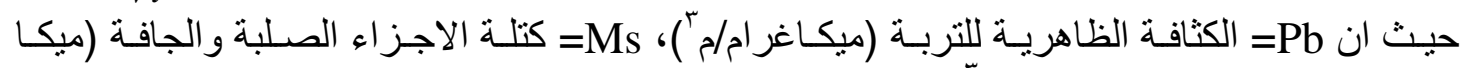

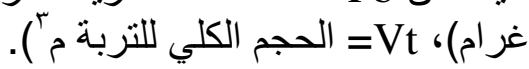
$\mathrm{f}=\left[1-\frac{P b}{P s}\right] \times 100$

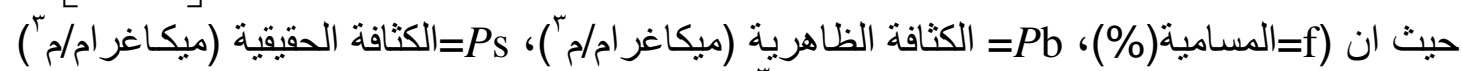

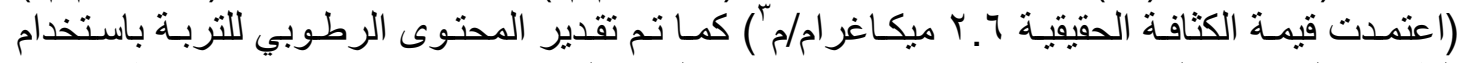

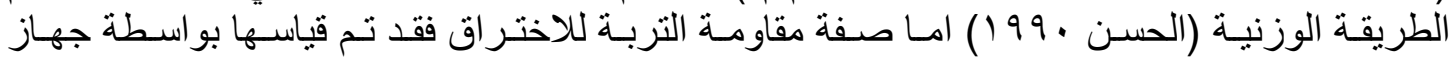

المخر اق الحقلي (البنترومينر).

\section{النتائج والمناقشة النجي}

يبين الجدول (T) ان المحراث القرصي الراسي سجل انخفاضا معنويا عن المحر اثين الاخرين

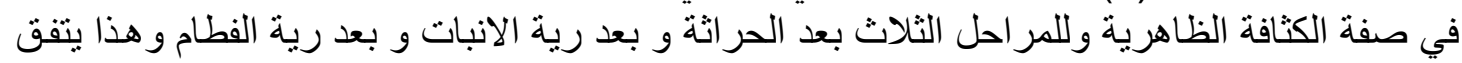

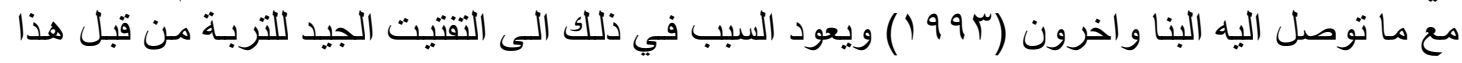

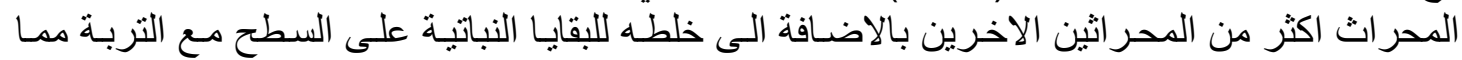

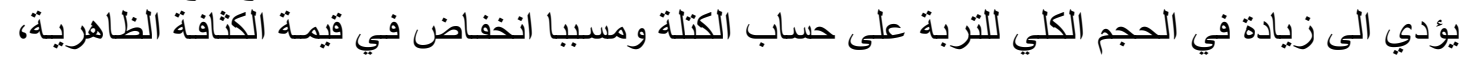

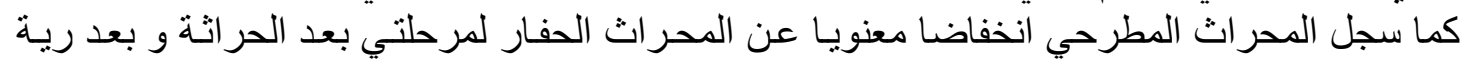

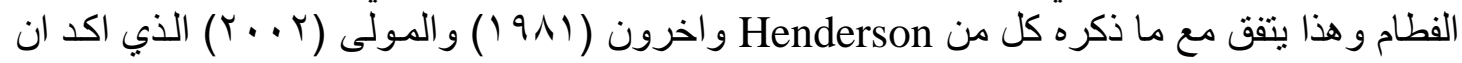

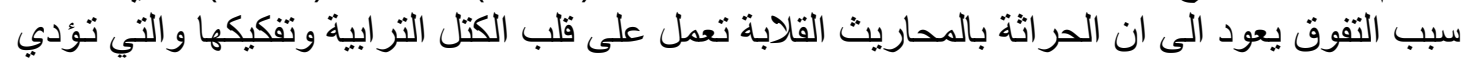

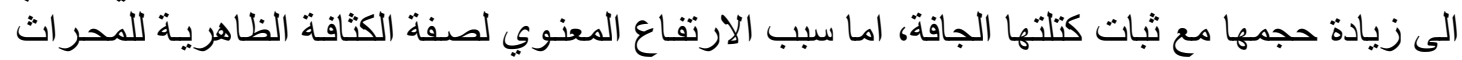
الحفار فيعود الى انه محر اث يعمل على تفكيك موضعي وتفنيت نسبي دون ان يكون هنالك قلب للمقطع 
و وؤديا الى قلع الحشائش و البقايا النباتية لتصبح ظاهرة بشكل جزئي او كامل فوق سطح التربـة المعامل

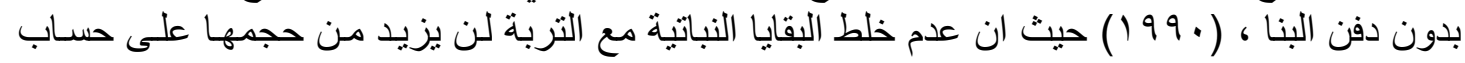

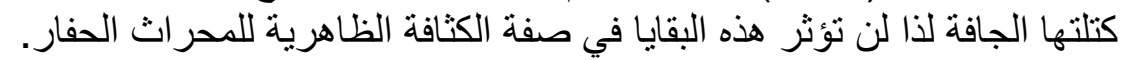

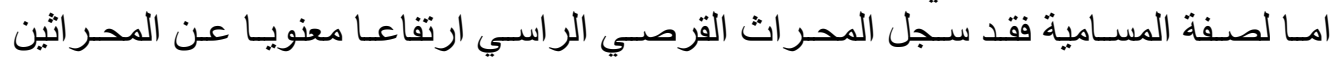

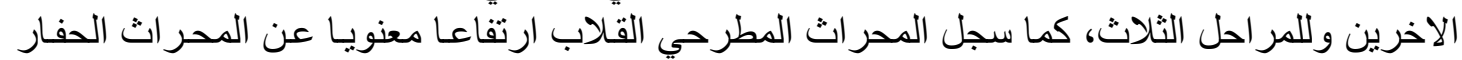

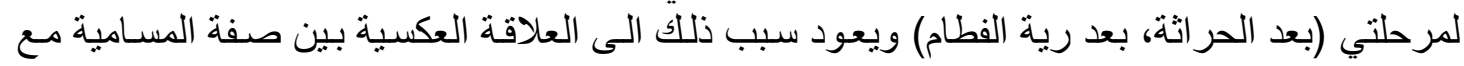

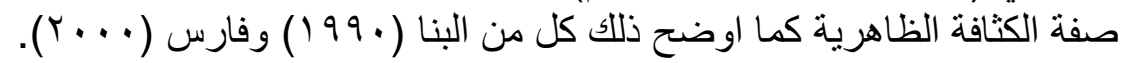

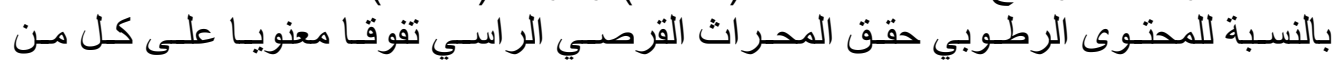

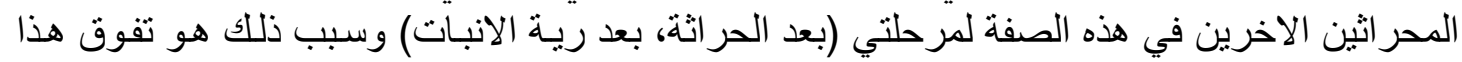

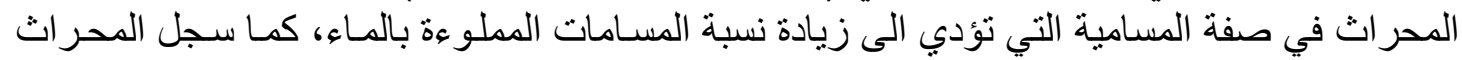

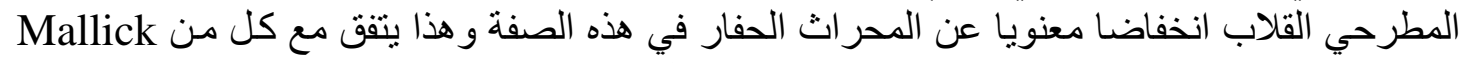

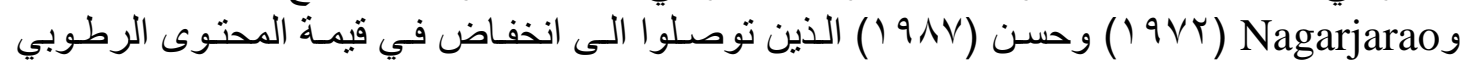

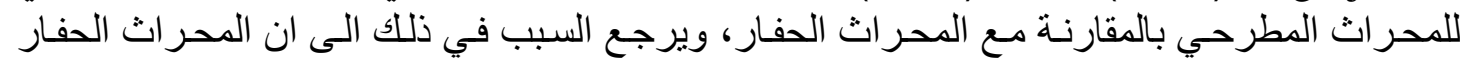

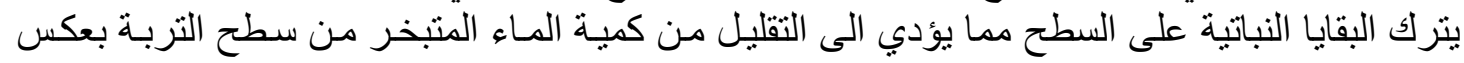

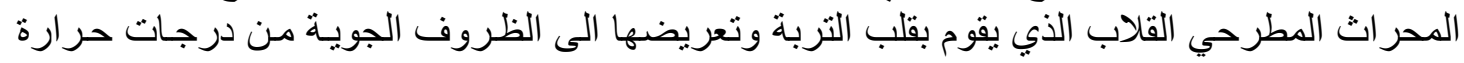

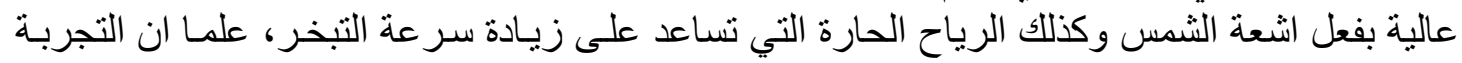

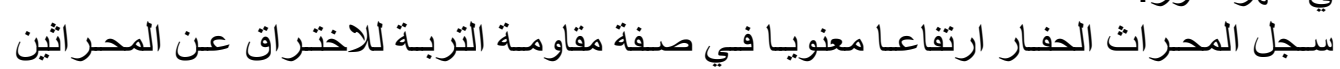

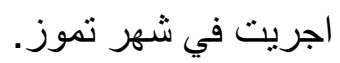

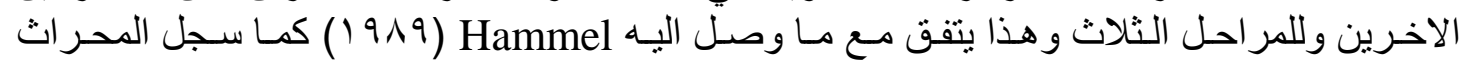

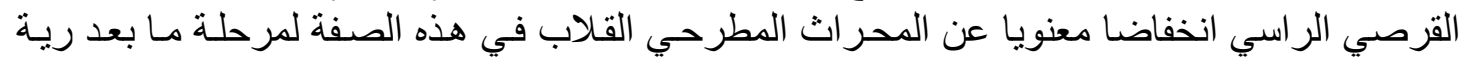

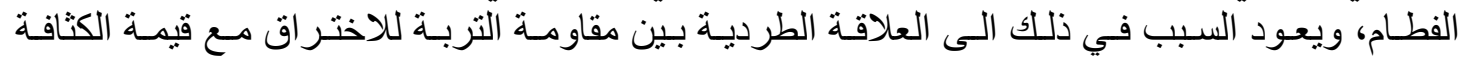

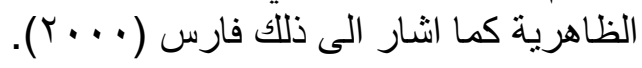

الجدول (r) : تأثثير أنواع المحاريث في الصفات الفيزيائية للتربة

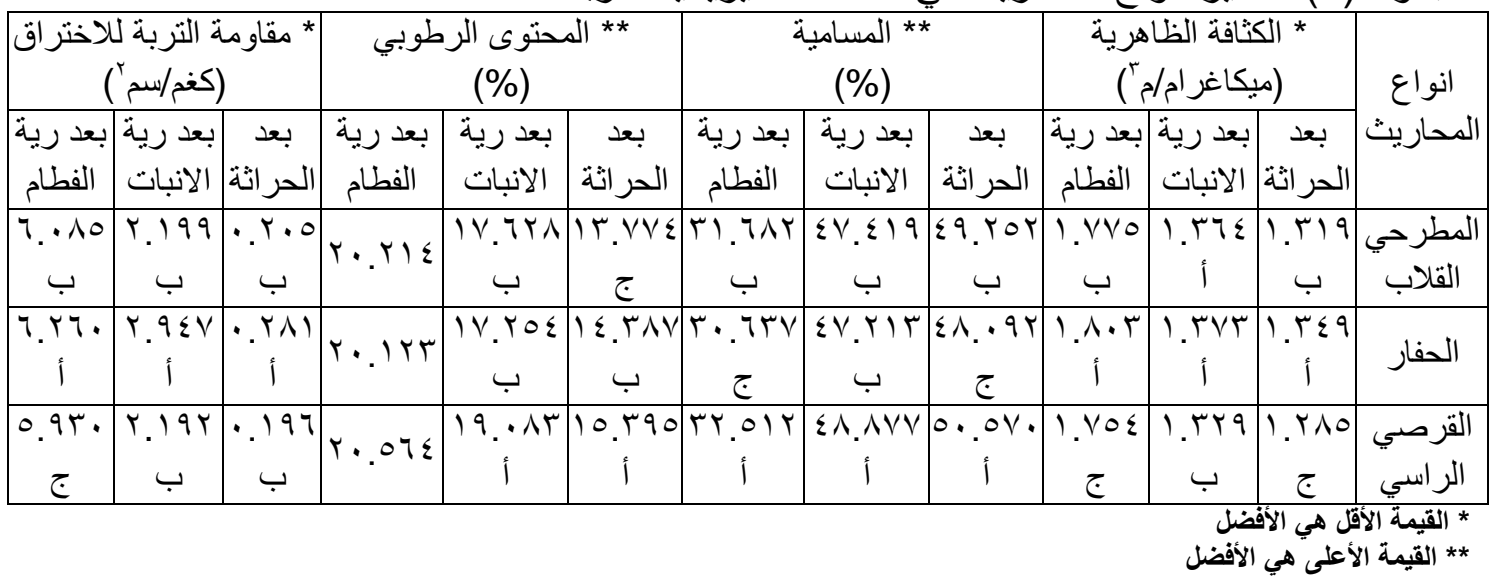

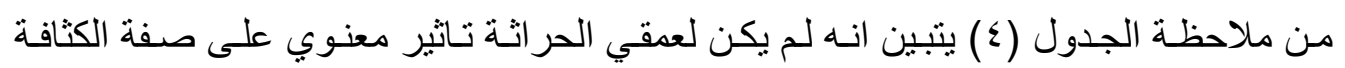

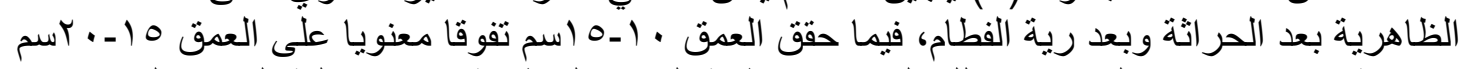

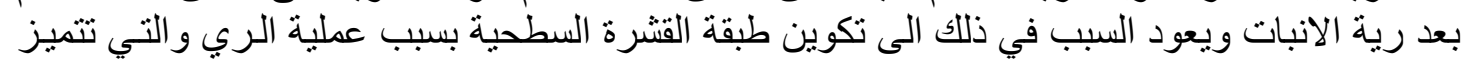

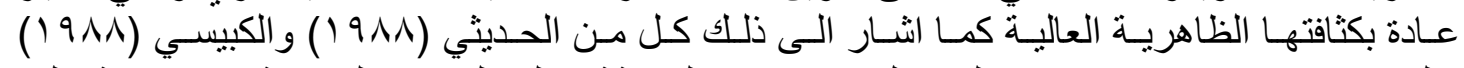

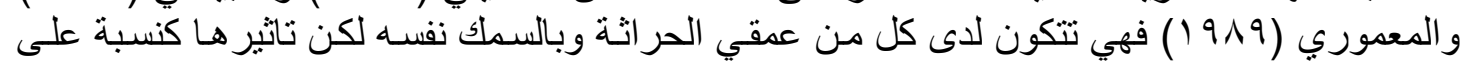

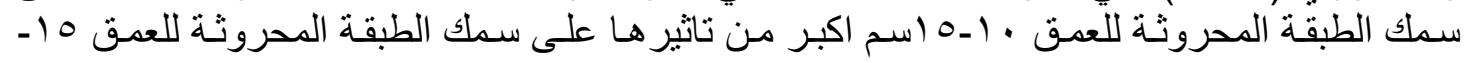

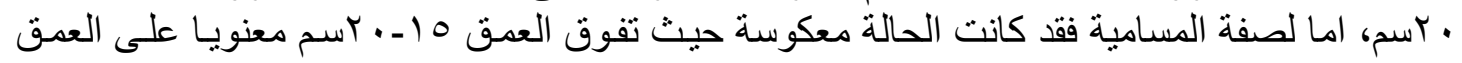

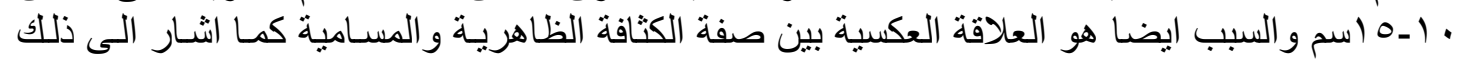




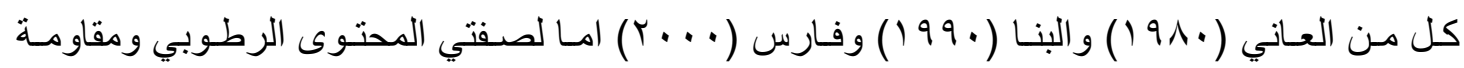

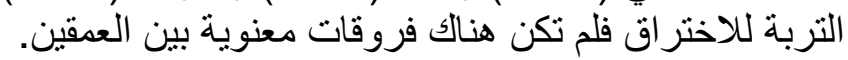

\begin{tabular}{|c|c|c|c|c|c|c|c|c|c|c|c|c|}
\hline & & & & & للتربة & لفيزيائية & لصفات | & ـاثة في & اق الحر & ناثير اعم & ( : & الجدول \\
\hline ) لاختراق & 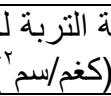 & * مقاوم * & طوبي & 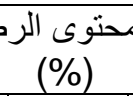 & di ** & & $\begin{array}{c}\text { (\%) } \\
\text { (\% }\end{array}$ & & برية & كيكافة الظاد & & اعماق \\
\hline |بعد رية & بلانبات رية & | الحراثة & الفطام رية & | بعد رية & الحر اثة & القطد رية & بلعد رية & الحر اثية & الفطام رية & | بعد رية & الحر اثة & الحم) \\
\hline 7.911 & $r .0 \leqslant 0$ & $\cdot r Y V$ & $r \cdot r \wedge 0$ & IV.AOT & $1 \leqslant . \Gamma_{0}$ & $r 1 . \varepsilon r v$ & $\begin{array}{c}\varepsilon V . r \leqslant 1 \\
\varphi\end{array}$ & $\leqslant q . \leqslant \vee N$ & 1.VAr & 1.479 & I.r. & $10_{-1}$. \\
\hline $1 . \wedge \varepsilon$ & r. $\leqslant \wedge 1$ & $\because r Y q$ & $r \cdot . r 17$ & $11.1 K T$ & $1 \leq .7 \wedge 1$ & M. I. & $\begin{array}{c}\sum \Lambda . \varepsilon r Y \\
j\end{array}$ & $\leqslant 9.1 \% \psi \mid$ & $1 . V V T$ & $\begin{array}{c}1 . r \varepsilon 1 \\
\varphi\end{array}$ & $1, r K r$ & $r \cdot-10$ \\
\hline
\end{tabular}

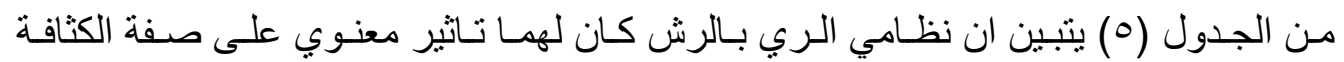

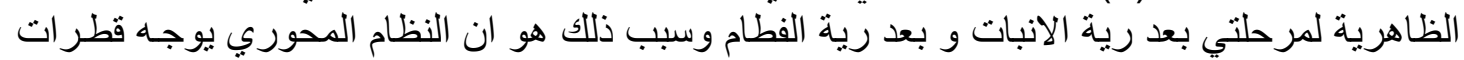

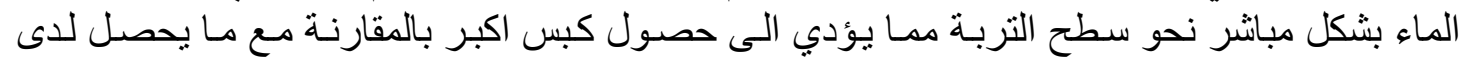

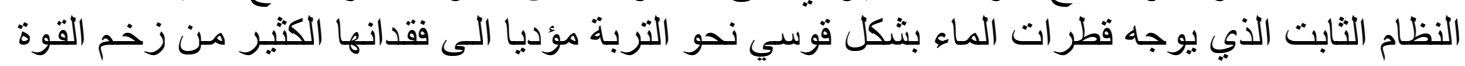

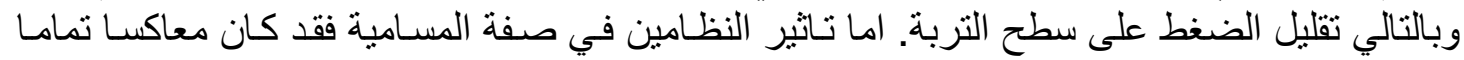

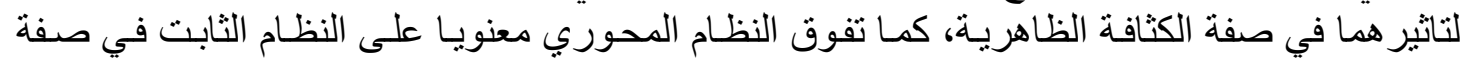

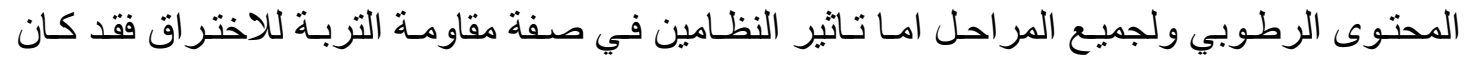
منو افقا تماما مع تاثير هما في صفي ولرئ الكثافة الظاهرية.

الجدول (0) تاثير انظمة الري في الصفات الفيزيائية للتربة

\begin{tabular}{|c|c|c|c|c|c|c|c|c|c|c|c|c|}
\hline \multicolumn{3}{|c|}{ * مقاومة التربة للاختر اق } & \multicolumn{3}{|c|}{$\begin{array}{c}\text { ** المحتوى الرطوبي } \\
\text { (\%) }\end{array}$} & \multicolumn{3}{|c|}{ 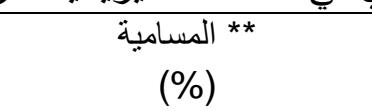 } & \multicolumn{3}{|c|}{ 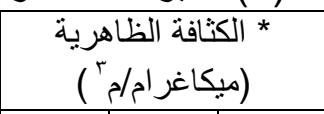 } & \multirow{2}{*}{ بالرشي } \\
\hline الفطام & | لبعد رية & الحر اثلة & بعد رية & | الانبات & الحراثة & بعد رية & الانبات & الحر اثثة & بعد رية & | لبعد رية & الحر اثة & \\
\hline $\begin{array}{l}0.900 \\
\end{array}$ & $\begin{array}{c}1.99 \mathrm{~V} \\
4\end{array}$ & $\cdot Y Y \Lambda$ & $\begin{array}{c}19 . \leqslant 1 . \\
4\end{array}$ & $\begin{array}{l}17.099 \\
ب\end{array}$ & . & $r_{i}^{r} \cdot{ }_{i}^{\wedge}$ & $\begin{array}{c}\sum \Lambda .9 T Y \\
i\end{array}$ & $\leqslant q . r q 4$ & $\begin{array}{l}\text { l. } \\
\\
\end{array}$ & (1.rT & $1 . \% 10$ & الثابت \\
\hline $\begin{array}{l}r . r Y V \\
1\end{array}$ & $r \cdot i_{i}$ & $\because K r V$ & $\underset{1}{Y 1}$ & 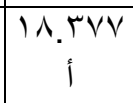 & $\sum_{1}^{1 \varepsilon . V O A}$ & ( & $\begin{array}{c}\sum 7 . \vee 01 \\
\varphi\end{array}$ & $\{9 ., \backslash V$ & $\begin{array}{c}1 . \vee \wedge \varepsilon \\
1\end{array}$ & $\begin{array}{c}1 . r 10 \\
1\end{array}$ & $1 . \pi r$. & لدحوري \\
\hline
\end{tabular}

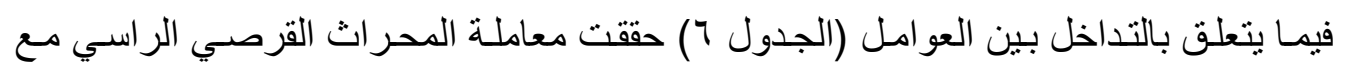

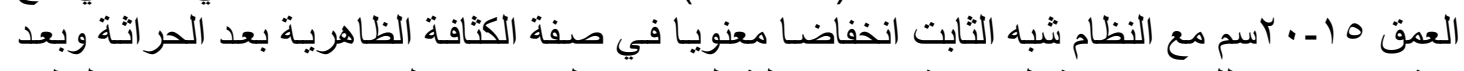

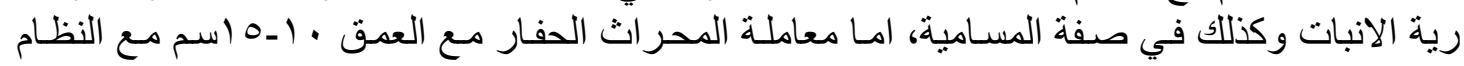

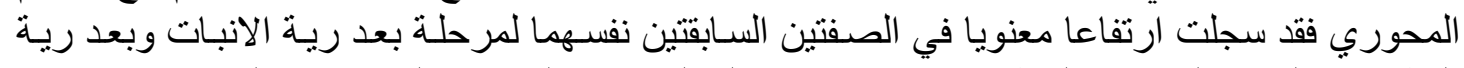

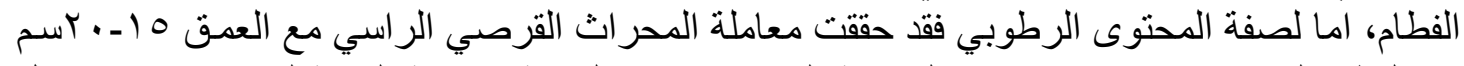

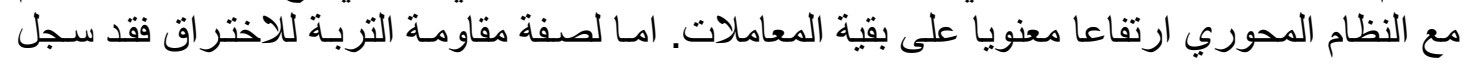

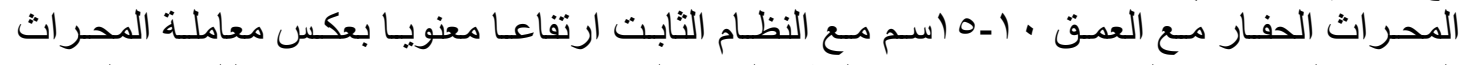

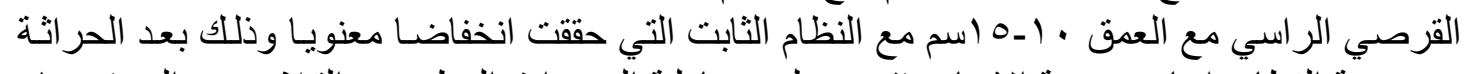

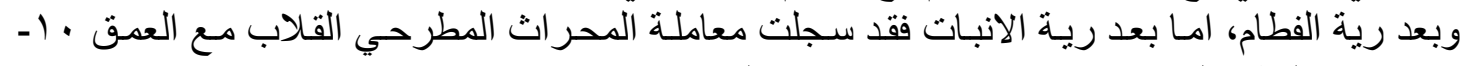

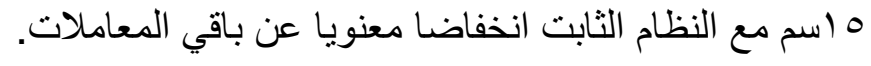


الجدول (؟) : تأثثر التداخل بين أنواع المحاريث وأعماق الحر اثنة وأنظمـة الري في الصفات الفيزيائية

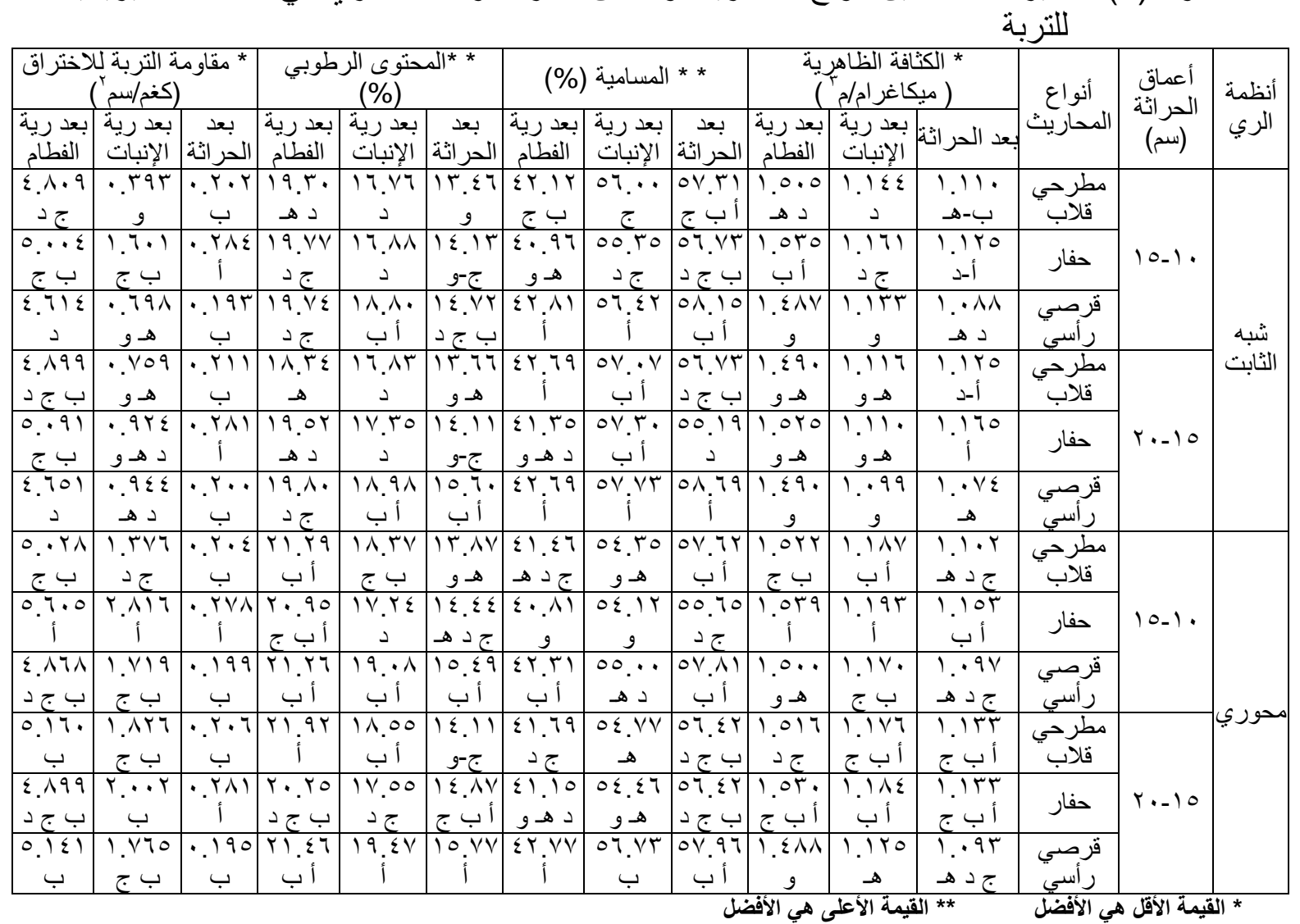

\title{
EFFECT OF SOME PLOW TYPES ON SOME SOIL PHYSICAL PROPERTIES UNDER SPRINKLER IRRIGATION SYSTEMS
}

Yassen H. Al-Tahan Ahmed M.A. Al-Ali Khan Baq

Agric. Mechanization Dept., College of Agric. and Forestry, Univ. of Mosul, Iraq

\begin{abstract}
This study was conducted in telefar region, Mosul governorate during agriculture season 2002-2003 to study the effect of three plow types (mold board, Chisel and one way disk) with two plowing depths (10-15 cm) and (15$20 \mathrm{~cm}$ ) and two sprinkler irrigation system on soil physical properties (bulk density, porosity, moisture content and soil penetration resistance) randomized complete block design (split - split plot design) was used and the results analyzed statistically. The results showed that the one way disk plow with the solid set sprinkler irrigation system surpassed in bulk density and porosity after plowing. The same plow with the two studied depth and the same irrigation system after germination irrigation and final irrigation surpassed in bulk density and porosity as well as soil penetration resistance characteristics. In moisture content of the soil the one way disk plow with depth of plowing $(15-20) \mathrm{cm}$ and the a center pivot sprinkler irrigation system significantly surpassed after plowing and after germination irrigation. After the final irrigation, the moldboard plow and the same plowing depth and the same irrigation system surpassed in moisture content characteristic.Also the
\end{abstract}


moldboard plow with the plowing depth (10-15) $\mathrm{cm}$ with irrigation system surpassed in soil penetration resistance .

\section{المصادر}

البنا، عزيز رمو (.999) (19. معدات تهيئة التربة، وزارة التعليم العالي والبحث العلمي، جامعة الموصل،

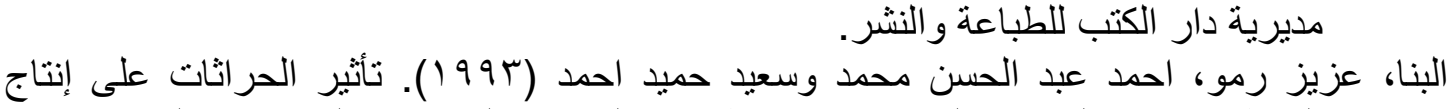

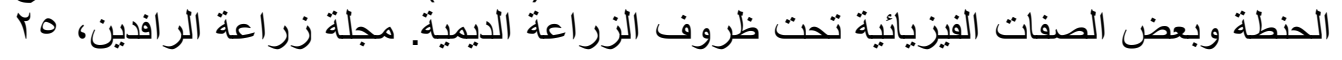

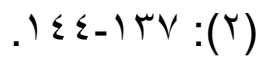

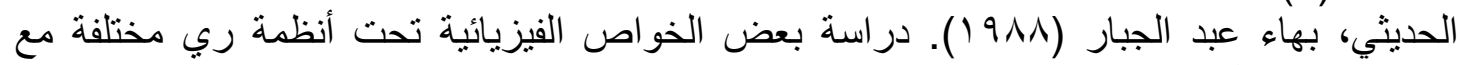

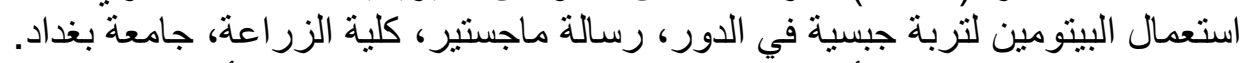

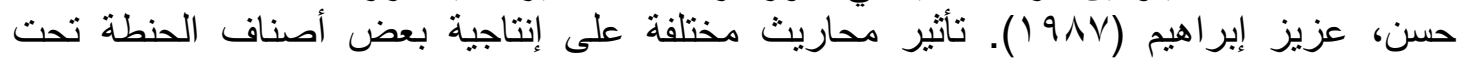

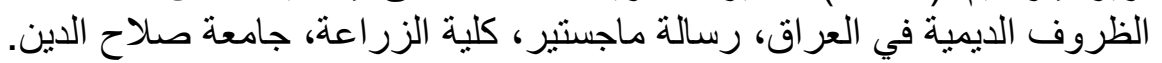

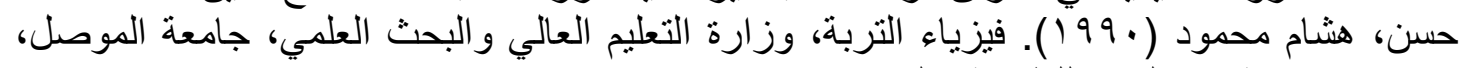

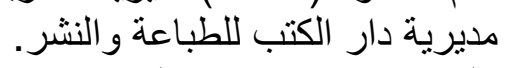

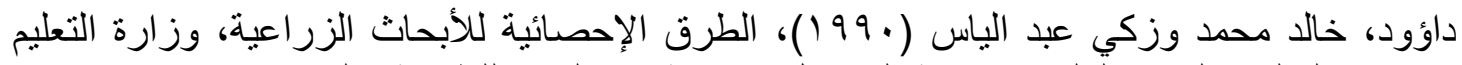

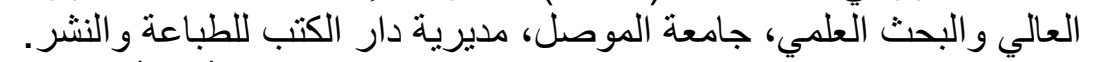

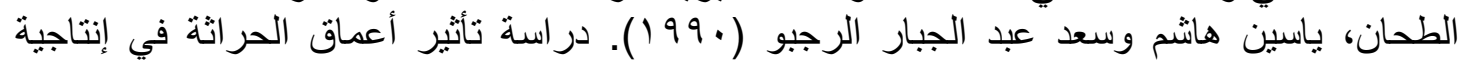

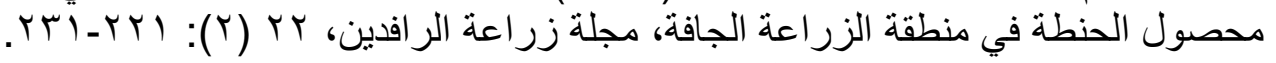

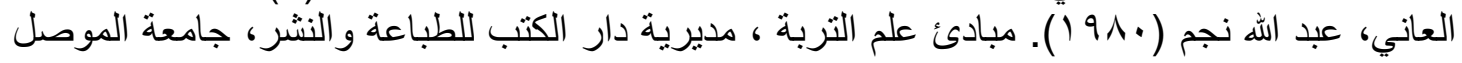

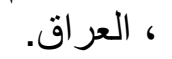

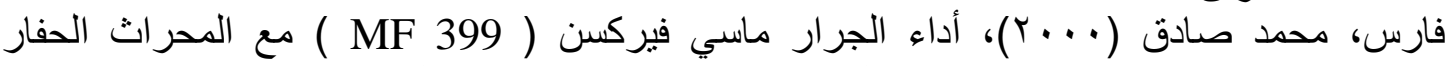

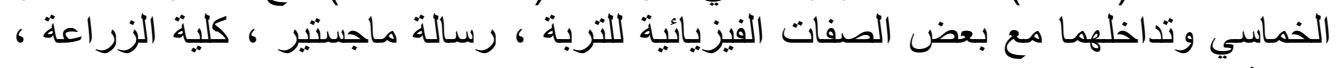

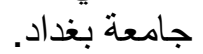

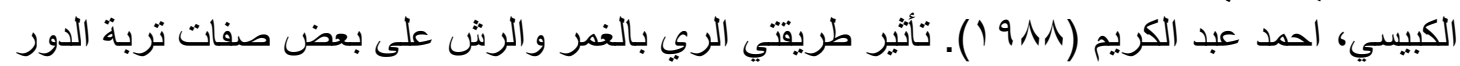

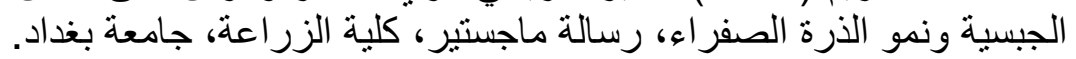

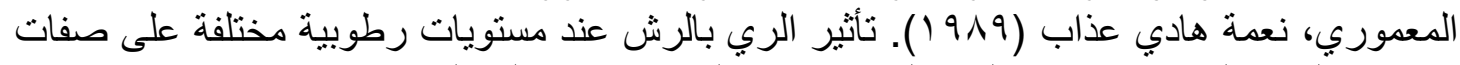

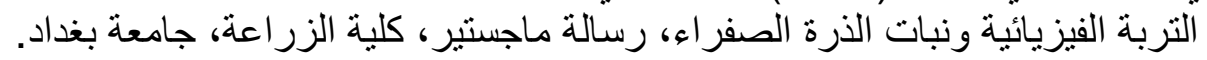

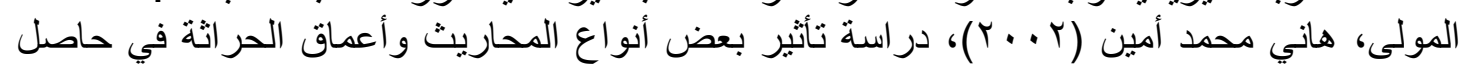

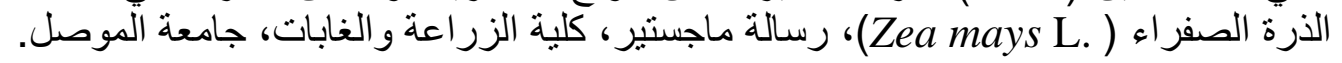
Hammel, J.E.(1989). Long term and crop rotation effects on bulk density and soil impedance in Northern Idaho. Soil Sci. Sco. Am.J.,53(6):15151519.

Mallick,S. and Y. Nagarajarao (1972). Effect of tillage on structure and plant growth under rainfed conditions. Indian J. of Agric. Sci., 42 (2): 827-831.

Henderson, H.D.; M.Almassi; A.A.Malik and Z. Mojaddadi (1981). Deep tillage in the bega' a valley, Lebanon.trans . of the ASAE, 24(6): 14661470. 